\title{
SOME CLASSES OF ORTHOGONAL POLYNOMIALS ASSOCIATED WITH MARTINGALES
}

\author{
PHILIP FEINSILVER
}

\begin{abstract}
The classes of orthogonal polynomials which arise as iterated stochastic integrals of a process with stationary independent increments are discussed. They are classes of Meixner polynomials.
\end{abstract}

This study was motivated by the well-known fact [7] that the iterated stochastic integrals of Brownian motion are given by Hermite polynomials $H_{n}(B(t), t)$. The question arises whether a similar phenomenon holds for other processes with stationary independent increments-do their iterated stochastic integrals yield classes of orthogonal polynomials?

There are two main components involved in the answer. One aspect is the theory of stochastic integrals-work inspired by Meyer [10, especially pp. 303-307, 318-320]. Two theorems are critical:

(1) LIN's THEOREM [8]. The generating function for the iterated stochastic integrals is the exponential martingale of Doléans-Dade.

(2) EMERY'S THEOREM (e.g. [4]). In the continuous case, the exponential martingale can be computed via a suitable discretization procedure. (See his Theorem 2, p. 261, with $e(x)=1+v x$, cf. below.)

The second aspect is the intimate relationship of the Meixner polynomials with the representation theory of the Heisenberg group (see $[5,6])$. The main feature here is that the lowering (pseudo-differential) operator $V(d / d x)$ satisfies a Riccati equation (cf. [5, 9]). The lowering operator $V$ acts on the polynomial sequence $J_{n}(x)$ by $V J_{n}=n J_{n-1}$ and the polynomials $J_{n}$ are given as $\xi^{n} 1$, where $V$, $\xi$ form a canonical pair of boson operators, i.e., their commutator $[V, \xi]=1$. In other words, the class of orthogonal polynomials arising in this study are identical to those that arise as Fock spaces in one variable-the function 1 plays the role of the vacuum $\Omega$, $V$ is the annihilation operator, $\xi$ the creation operator (cf. [6]).

Thanks to the theorems mentioned above, we consider the discrete case first, then take limits to deal with the continuous case. We begin with a probability measure

Received by the editors August 9, 1985. Presented at the AMS meeting, Notre Dame, April 1984; also to the Mathematics Seminar (Meyer) at Strasbourg, May 1985.

1980 Mathematics Subject Classification (1985 Revision). Primary 60G46; Secondary 33A65, 60H05. 
$p(d x)$ on $\mathbf{R}$ satisfying

$$
\int_{\mathbf{R}} e^{i \xi x} p(d x)=e^{L(i \xi)}, \quad \xi \in \mathbf{R},
$$

where we assume that the function $L$ has an analytic extension to a neighborhood of 0 in C. We denote this extension by $L(z), z \in$ C. E.g., if $p(d x)=$ $\left(\exp \left(-x^{2} / 2\right) / \sqrt{2 \pi}\right) d x$, then $L(z)=z^{2} / 2$. The convolution powers of $p$ are denoted by $p_{t}$, where in the discrete case $t \in \mathbf{N}$. In the continuous case $L$ must be of Lévy-Khinchine type; no such restriction applies in the discrete case. (Note. In the sequel we use the notation $p(d x)=p(x) d x$, in the sense of distributions.)

I. Rodrigues formulae. In this case we consider the process $S_{n}=X_{1}+X_{2}$ $+\cdots+X_{n}$, where the $X_{j}$ are independent, mean zero, with distribution $p$. The exponential martingale is

$$
E_{n}(v)=\prod_{j=1}^{n}\left(1+v X_{j}\right)=\sum_{k=0}^{n} v^{k} I_{k} .
$$

The $I_{k}$ are discrete iterated sums, readily recognized as the elementary symmetric functions in the variables $X_{1}, X_{2}, \ldots, X_{n}$. The calculation, \langle\rangle denoting expected value, $\sigma^{2}=\left\langle X_{j}^{2}\right\rangle$,

$$
\langle E(v) E(w)\rangle=\prod_{1}^{n}\left\langle 1+(v+w) X_{j}+v w X_{j}^{2}\right\rangle=\left(1+v w \sigma^{2}\right)^{n}
$$

shows that the $I_{k}$ are orthogonal, the cross terms vanishing.

In order to have orthogonal polynomials, these $I_{k}$ must be functions of the variable $x$, that is, of $S_{n}$ alone (besides $n$, of course). If this is the case we say that the process $S_{n}$ is observable-since the $I_{k}$ are functions only of the "physical parameters" $x=S_{n}$ and $t=n$, space and time.

THEOREM 1. The exponential martingale for an observable discrete process is of the form

$$
\mu(x, t)=p_{t}(x)^{-1}\left(1+v V^{*}\right)^{t} p_{t}(x)
$$

where the pseudo-differential operator $V^{*}(D)=V(-D)$ where $V$ has symbol $V(z)=$ $L^{\prime}(z)$.

REMARK. The equality stated means that $\mu(x, t) p(x)=\left(1+v V^{*}\right)^{t} p(x)$ in the sense of distributions. (Note. $D=d / d x$.)

Proof. The key notion is that for the observable case since $E_{n}(v)$ is only a function of $x=S_{n}$ and $t=n$ it must equal the conditional expectation

$$
\mu(x, t)=E\left(E_{n}(v) \mid S_{n}=x\right)=\left\langle E_{n} \delta\left(S_{n}-x\right)\right\rangle / p_{n}(x) .
$$

Using the Fourier representation $\delta(y)=\int_{\mathbf{R}} e^{i \xi y} d \xi / 2 \pi$,

$$
\mu(x, t) p_{t}(x)=\int\left\langle\prod_{j}\left(1+v X_{j}\right) e^{i \xi X_{j}}\right\rangle e^{-i \xi x} d \xi / 2 \pi .
$$


Consider a single factor with $X$ replacing an $X_{j}$,

Thus, with $D$ denoting $d / d x$,

$$
\begin{aligned}
\left\langle(1+v X) e^{i \xi X}\right\rangle & =\left(1+\frac{v}{i} \frac{\partial}{\partial \xi}\right)\left\langle e^{i \xi X}\right\rangle \\
& =\left(1+\frac{v}{i} \frac{\partial}{\partial \xi}\right) e^{L(i \xi)} \\
& =\left(1+v L^{\prime}(i \xi)\right) e^{L(i \xi)} .
\end{aligned}
$$

$$
\begin{aligned}
\mu(x, t) p_{t}(x) & =\int_{\mathbf{R}}\left(1+v L^{\prime}(i \xi)\right)^{n} e^{n L(i \xi)} e^{-i \xi x} d x / 2 \pi \\
& =\left(1+v L^{\prime}(-D)\right)^{n} \int_{\mathbf{R}} e^{-i \xi x} e^{n L(i \xi)} d \xi / 2 \pi \\
& =\left(1+v V^{*}\right)^{t} p_{t}(x)
\end{aligned}
$$

as required.

THEOREM 2. The exponential martingale for an observable continuous process is of the form

$$
\mu(x, t)=p_{t}(x)^{-1} e^{v t V^{*}} p_{t}(x)
$$

with $V(z)=L^{\prime}(z)$.

Proof. By the theorems mentioned above, we consider the process $S(t)=X_{1}+$ $X_{2}+\cdots+X_{n}$ where the $X_{j}$ are independent, mean zero, with distribution $p_{t / n}$, i.e.

$$
X_{j}=S(j t / n)-S((j-1) t / n), \quad S(0)=0 .
$$

The discrete approximant satisfies

$$
\mu_{n}(x, t)=\left\langle\mu_{n} \delta(S(t)-x)\right\rangle / p_{t}(x) .
$$

As above, we find

$$
\mu_{n}(x, t) p_{t}(x)=\left(1+v t V^{*} / n\right)^{n} p_{t}(x) .
$$

In the limit as $n \rightarrow \infty$ we arrive at the stated form.

Now, expanding the generating functions we have the iterated stochastic integrals expressed via the Rodrigues-type formulae

$$
I_{k}(x, t)=c_{k} p_{t}(x)^{-1} V^{* k} p_{t}(x)
$$

with suitable constants $c_{k}$.

II. Riccati equation. The next step is to require that the $I_{k}$ be in fact polynomials in $x, t$.

LEMMA 1. Let $\eta_{k}(z)=e^{-t L(z)}(\partial / \partial z)^{k} e^{t L(z)}$. Then $\eta_{k}(i \xi) e^{t L(i \xi)}$ is the Fourier transform of $x^{k} p_{t}(x)$. That is, as operators, the $\eta_{k}$ act according to $\eta_{k}^{*} p_{t}(x)=x^{k} p_{t}(x)$.

Proof. Taking Fourier transforms, using the standard relations

$$
\left(x^{k} f(x)\right)^{\swarrow}(\xi)=\left(\frac{1}{i} \frac{\partial}{\partial \xi}\right)^{k} f^{\check{ }}(\xi)
$$

yields the result, with $\check{f^{\prime}}(\xi)=\int_{\mathbf{R}} e^{i \xi x} f(x) d x$. 
THEOREM 3. For the $I_{k}(x, t)$ to be polynomials, the function $V(z)$ must satisfy $a$ differential equation of the form $V^{\prime}=a+b V+c V^{2}$ for suitable constants $a, b, c$.

Proof. For $k=1, \eta_{1}(z)=t L^{\prime}(z)=t V(z)$. For $k=2, \quad \eta_{2}(z)=t L^{\prime \prime}(z)+$ $\left(t L^{\prime}(z)\right)^{2}=\alpha V^{\prime}+\beta V^{2}$, say. The polynomial $I_{2}(x, t)$ satisfies

$$
c I_{2}(x, t) p_{t}(x)=V^{* 2} p_{t}(x)
$$

or, by the lemma, there exist constants $\lambda, \mu, \nu$, and $\lambda_{j}$ such that

$$
V^{2}=\lambda+\mu \eta_{1}+\nu \eta_{2}=\lambda_{1}+\lambda_{2} V+\lambda_{3} V^{\prime}+\lambda_{4} V^{2}
$$

If $\lambda_{3}=0$, then the function $V(z)$ would satisfy a quadratic equation. The centering of the process gives $V(0)=L^{\prime}(0)=0$ and thus $V$ would be constant. Hence $\lambda_{3} \neq 0$ and the result follows.

REMARK. Notice that only $I_{1}$ and $I_{2}$ play an explicit role, cf. [1].

III. Conclusion. It now follows that the polynomials are in fact Meixner polynomials (cf. [2, 6, 9]). That is, the processes are of the types: binomial, negative binomial, gamma, Poisson, or Gaussian. Briefly, we may say these are of "Bernoulli type."

It is interesting to see how in the Bernoulli case the two-valuedness leads to observability. Consider $X_{j}$ taking values 1 and $0, P\left(X_{j}=1\right)=p$. Let $\nu=$ number of $X_{j}$ equalling $0, \pi=$ number of the $X_{j}$ equalling +1 . For the process $\sum_{1}^{n} X_{j}$ at position $x$ at time $t$, we have $x=\pi, t=\pi+\nu$. Form the centered increments $Y_{j}=X_{j}-p$. Thus,

$$
\begin{aligned}
E_{n}(v) & =\prod_{1}^{n}\left(1+v Y_{j}\right)=(1+v(1-p))^{\pi}(1-p v)^{\nu} \\
& =(1+v(1-p))^{x}(1-p v)^{t-x} .
\end{aligned}
$$

REMARK. A recent paper by Dunkl [3] discusses some Dirichlet problems on the disk. The Meixner-Pollaczek polynomials play a major role. They are of Bernoulli type with $L(z)=\log \cos \beta-\log \cos (z+\beta)$ (see [3, p. 170]). They correspond to infinitely divisible laws.

ACKNowledgment. The author would like to extend his thanks to Professor R. Schott of Nancy and Professors Meyer, Bakry, and Emery of Strasbourg for their fine hospitality and encouragement of this work.

The paper is based on a talk presented at the AMS meeting in Notre Dame, April 1984; the special session was graciously chaired by Professor Chihara.

\section{REFERENCES}

1. M. Arbib, Hitting and martingale characterizations of one-dimensional diffusion, Z. Wahrsch. Verw. Gebiete 4 (1965), 232-247.

2. T. S. Chihara, An introduction to orthogonal polynomials, Math. Appl., No. 13, Gordon and Breach, 1978.

3. C. F. Dunkl, Orthogonal polynomials and a Dirichlet problem related to the Hilbert transform, Indag. Math. 47 (1985), 147-171.

4. M. Emery, Stabilité des solutions des équations différentielles stochastiques, application aux intégrales multiplicatives stochastiques, Z. Wahrsch. Verw. Gebiete 41 (1978), 241-262. 
5. P. 'Feinsilver, Special functions, probability semigroups and Hamiltonian flows, Lecture Notes in Math., vol. 696, Springer-Verlag, Berlin and New York, 1978.

6. Canonical representation of the Bernoulli process, in Probability Measures on Groups, Lecture Notes in Math., vol. 928, Springer-Verlag, Berlin and New York, 1982, pp. 90-95.

7. T. Hida, Brownian motion, Springer-Verlag, Berlin and New York, 1980.

8. T. F. Lin, Multiple integrals of a homogeneous process with independent increments, Ann. Probab. 9 (1981), 529-532.

9. J. Meixner, Orthogonale Polynomsysteme mit einem besonderen Gestalt der erzeugenden Funktion, J. London Math. Soc. 9 (1934), 6-13.

10. P. Meyer, Lecitures on stochastic integrals, in Séminaire des Probabilités X, Lecture Notes in Math., vol. 511, Springer-Verlag, Berlin and New York, 1976, pp. 303-320.

Department of Mathematics, Southern Illinois University, CaRbondale, Illinois 62901 